\title{
Study on flowering characteristics and categorization of rose cultivars for color, fragrance and usage
}

\author{
S. Shahrin ${ }^{a}$, M. Z. K. Roni ${ }^{a}$, T. Taufique ${ }^{a}$, H. Mehraj ${ }^{b}$ and A. F. M. Jamal Uddin ${ }^{a}$ \\ aept. of Horticulture, Sher-e-Bangla Agricultural University, Dhaka-1207, Bangladesh \\ ${ }^{b}$ The United Graduate School of Agricultural Sciences, Ehime University, 3-5-7 Tarami, Matsumaya, Ehime \\ 790-8556, Japan
}

\begin{abstract}
An experiment was conducted to evaluate the performance of rose cultivars and their categorization based on color, fragrance and use. Field experiment included forty four rose cultivars coded from $V_{1}$ to $V_{44}$. It was found that all rose cultivars varied significantly for the studied characters like number of shoots/plant, leaf area, chlorophyll content, number of flower/plant and petals per flower. Rose cultivars were categorized in nine groups in accordance to color, three groups in accordance to the fragrance and two groups in accordance usage excluding the wild type. $V_{3}$ (Tajmahal), $V_{4}$ (Yellow star), $V_{14}$ (Lavender gold), $V_{16}$ (Compassion), $V_{21}$ (SAU hero), $V_{23}$ (Yellow gold), $V_{25}$ (Sleepy moon), $V_{26}$ (Sweet doll), $V_{30}$ (Chrysanthemum rose) and $V_{35}$ (Sweet sakata) cultivars were found as the cut flowers and rest were pot/bedding flower. Among the 44 cultivars desired one could be isolated easily for the specific purpose. Roses are vegetatively propagated and they are selected for defined traits such as stem length, flower bud, flower number, flower size and flower qualities (shape, color, fragrance). Breeding to modify a single characteristic generally results in changes to other characters as well.
\end{abstract}

Keywords: Rose, flowering characteristics, categorization and usage

Please cite this article as: Shahrin, S., Roni, M. Z. K., Taufique, T., Mehraj, H. \& Jamal Uddin, A. F. M. (2015). Study on flowering characteristics and categorization of rose cultivars for color, fragrance and usage. Journal of Bioscience and Agriculture Research 04(01): 20-30.

This article is distributed under terms of a Creative Common Attribution 4.0 International License

\section{Introduction}

Garden rose (Rosa hybrida hort.) is one of the most intensively used and most diverse flowering ornamental shrubs in the world. Bangladesh has a great potential for rose production. Growth, flowering and yield varied significantly due to the variation of variety in gerbera (Singh and Ramachandran, 2002; Sankar et al., 2003; Mahanta et al., 2003; Reddy et al. 2003), tomato (Gautam et al., 2013; Olaniyi et al., 2010) due to their genetic variation (Hammad, 2009). Varietal performance was also studied in rose by Ranchana et al. (2014) and significant variations were observed in each cultivar for length and number of petals per flower, number of prickles, fragrance, flower persistence life and color, bush shape and overall performance with respect to climatic conditions (Nadeem et al., 
2011). Color and fragrance are the most desirable traits of roses and consumer demand varies in color and fragrance. Two scented parents can produce an unscented or unpleasantly scented offspring (Cherri-Martin et al., 2007) while for the production of a new color offspring by breeding it is important to know the color of the parent. Roses are grown by commercial flower growers for the cutflower market and for domestic and industrial landscaping, for use as ornamentals, as well as for the food and medicinal value. Morphological variables of a set of germplasm or cultivars were determined to provide information for breeders (Mehraj et al., 2014a). Color variability and growth habit diversification of the garden rose make it almost impossible to compare its varieties using traditional methods. From the breeders experience it can emphasize to categorize the rose cultivars as they can easily select the parent to produce new offspring with attractive foliage, outstanding colors and shapes and with good fragrance. The aim of the study was to evaluate the performance of rose cultivars and their categorization for color, fragrance and usage.

\section{Materials and Methods}

Location and period of the experiment: An experiment was conducted at rose garden, Horticulture farm at the Sher-e-Bangla Agricultural University, Dhaka, Bangladesh during October 2012 to March 2013.

Genetic materials: Experiment consisted 44 rose cultivars viz. $\mathrm{V}_{1}$ : Wild rose; $\mathrm{V}_{2}$ : Crazy love bicolor; $\mathrm{V}_{3}$ : Tajmahal; $\mathrm{V}_{4}$ : Yellow star; $\mathrm{V}_{5}$ : Ekuse lal; $\mathrm{V}_{6}$ : Afrodita; $\mathrm{V}_{7}$ : black divorce; $\mathrm{V}_{8}$ : Attarcted red; $\mathrm{V}_{9}$ : Missing love; $\mathrm{V}_{10}$ : Pinky smile; $\mathrm{V}_{11}$ : Sweet love; $\mathrm{V}_{12}$ : Red baby; $\mathrm{V}_{13}$ : Star fire; $\mathrm{V}_{14}$ : Lavender gold; $\mathrm{V}_{15}$ : Lemon star; $\mathrm{V}_{16}$ : Compassion; $\mathrm{V}_{17}$ : Charming lady; $\mathrm{V}_{18}$ : Dream bangla; $\mathrm{V}_{19}$ : Printed moon; $\mathrm{V}_{20}$ : Fire and ice; $V_{21}$ : SAU hero; $V_{22}$ : SAU prince; $V_{23}$ : Yellow gold; $V_{24}$ : Ocean heart; $V_{25}$ : Sleepy moon; $V_{26}$ : Sweet doll; $V_{27}$ : Moon light; $V_{28}$ : Pain blue; $V_{29}$ : Ruin night; $V_{30}$ : Chrysanthemum rose; $V_{31}$ : Mystery love; $V_{32}$ : SAU king; $V_{33}$ : Ice barg; $V_{34}$ : Yellow erecta; $V_{35}$ : Sweet sakata; $V_{36}$ : Anindo kun hazari; $V_{37}$ : Yellow papiliom; $\mathrm{V}_{38}$ : Red eye; $\mathrm{V}_{39}$ : Sympathy; $\mathrm{V}_{40}$ : Mini master; $\mathrm{V}_{41}$ : Pretty girl; $\mathrm{V}_{42}$ : Sad 23; $\mathrm{V}_{43}$ : Aranchan and $\mathrm{V}_{44}$ : Sweet mum (Plate 1). Three years old rose plants were selected for the experiment. Plants were pruned at 13 October, 2012.

Experimental design and data collection: Experiment was followed in Randomized Completely Block Design with three replications. Data were collected on number of shoots/plant, number of leaves $/ 10 \mathrm{~cm}$ shoot, number of thorns $/ 10 \mathrm{~cm}$ shoot, leaf area, chlorophyll content, days to flower bud initiation, number of flower/plant and petals per flower. Leaf area was measured by using CL-202 leaf area meter in non-destructive method. Chlorophyll content was measured by using SPAD-502.

Statistical analysis: Collected data were analyzed statistically using MSTAT-C computer package program and significance of the difference among the treatment means was estimated by the Least Significant Difference (LSD) test at 5\% level of significance (Gomez and Gomez, 1984).

\section{Results and Discussion}

Number of shoot per plant: Maximum number of shoot/plant was found from $V_{36}$ (42.0) followed by $\mathrm{V}_{27}$ (32.0) whereas minimum from $\mathrm{V}_{1}, \mathrm{~V}_{13}, \mathrm{~V}_{33}, \mathrm{~V}_{39}, \mathrm{~V}_{42}$ (4.0) (Table 01). Variation in number of shoots/plant was also observed previously in rose (Tabassum et al., 2002; Ramzan et al., 2014; Manjula, 2005; Paramagoudar, 2010), carnation (Gharge et al., 2011; Tarannum et al., 2014; Singh et al., 2013; Pralhad, 2009), china aster (Zosiamliana et al., 2012; Munikrishnappa et al., 2013), in french bean (Moniruzzaman et al., 2007) and tomato (Ahmad et al., 2007). The difference in number of shoots/plant among the cultivars might be due to influence of the genetic makeup of the cultivars.

Number of leaves per $10 \mathrm{~cm}$ shoots: Maximum number of leaves $/ 10 \mathrm{~cm}$ shoot was found from $V_{12}$ (7.0) followed by $V_{23}(6.0)$ whereas minimum from $V_{2}, V_{7}, V_{10}, V_{11}, V_{17}, V_{21}, V_{30}, V_{32}, V_{33}, V_{34}, V_{35}, V_{42}(3.0)$ which is statistically similar to $\mathrm{V}_{3}, \mathrm{~V}_{4}(3.3)$ (Table 01). Variation in number of leaves/ $10 \mathrm{~cm}$ shoot and leaves/plant was also observed previously in rose (Ahmad et al., 2011; Manjula, 2005; Paramagoudar, 2010), carnation (Gharge et al., 2011; Tarannum et al., 2014; Pralhad, 2009), gerbera (Hossain et al., 
2015; Mehraj et al., 2014b; Jamal Uddin et al., 2014a; Mahmood et al., 2013; Chobe et al., 2010; Kumari et al., 2010; Pattanashetti, 2009; Shruti et al., 2013), strawberry (Ahsan et al., 2014; Hossan et al., 2013), tomato (Biswas et al., 2014) and chilli (Mehraj et al., 2014c).

Density of thorns per $10 \mathrm{~cm}$ shoots: Maximum number of thorns $/ 10 \mathrm{~cm}$ shoot was found in $V_{20}$ (47) followed by $V_{9}, V_{28}(26)$ whereas minimum from $V_{4}, V_{14}, V_{15}, V_{29}, V_{30}, V_{37}, V_{41}$, and $V_{43}$ (0.3) (Table 01). Variation in number of thorns $/ 10 \mathrm{~cm}$ shoot was also observed previously in rose (Manjula, 2005).

Leaf area: Maximum leaf area was found from $V_{25}\left(69.5 \mathrm{~cm}^{2}\right)$ which is statistically similar to $V_{16}(68.4$ $\left.\mathrm{cm}^{2}\right)$ followed by $V_{33}\left(63.7 \mathrm{~cm}^{2}\right)$ whereas minimum from $V_{42}\left(23.5 \mathrm{~cm}^{2}\right)$ which is statistically similar to $\mathrm{V}_{1}\left(24.6 \mathrm{~cm}^{2}\right.$ ) (Table 01). Variation in leaf area was also observed previously in rose (Ahmad et al. 2011; Manjula, 2005; Paramagoudar, 2010), dendrobium (Mehraj et al., 2014e), gerbera (Chobe et al., 2010; Kumari et al., 2010; Pattanashetti, 2009; Shruti et al., 2013) and strawberry (Hossan et a., 2013).

Chlorophyll content: Maximum chlorophyll was found in $V_{35}(68.9 \%)$ followed by $V_{21}(61.2 \%)$ whereas minimum from $\mathrm{V}_{15}(35.0 \%)$ (Table 01). Variation in chlorophyll content was also observed previously in rose (Ahmad et al., 2011), dendrobium (Mehraj et al., 2014e), tomato (Mehraj et al., 2014d), bottle gourd (Jamal Uddin et al., 2014b), sweet gourd (Jamal Uddin et al., 2014c) and mustard (Datta et al., 2011).

Days to flower bud initiation: Maximum days were taken to flower bud initiation found in $V_{6}(40.0$ days) followed by $\mathrm{V}_{10}$ (36.0 days) whereas minimum from $\mathrm{V}_{16}$ (19.0 days) (Table 01). Variation in days to flower bud initiation was also observed previously in rose (Manjula, 2005; Paramagoudar, 2010), carnation (Maitra et al., 2013; Tarannum et al., 2014; Pralhad, 2009), china aster (Zosiamliana et al., 2012), lisianthus (Jamal Uddin et al., 2013), gerbera (Pattanashetti, 2009) and dendrobium (Mehraj et al., 2014e).

Numbers of flowers per plant: Maximum numbers of flowers/plant was found in $V_{43}$ (68.0) followed by $\mathrm{V}_{11}$ (20.0) whereas minimum from $\mathrm{V}_{3}, \mathrm{~V}_{9}, \mathrm{~V}_{13}, \mathrm{~V}_{21}, \mathrm{~V}_{22}, \mathrm{~V}_{25}, \mathrm{~V}_{33}, \mathrm{~V}_{39}, \mathrm{~V}_{42}$ (1.0) (Table 01). Variation in numbers of flowers/plant was also observed previously in rose (Tabassum et al., 2002; Ramzan et al., 2014; Paramagoudar, 2010), carnation (Gharge et al., 2011; Maitra et al., 2013; Singh et al., 2013), china aster (Munikrishnappa et al., 2013), gerbera (Mahmood et al., 2013; Chobe et al., 2010), lisianthus (Jamal Uddin et al., 2013), strawberry (Hossan et al., 2013) and in tomato (Nahiyan et al., 2014).

Numbers of petals per flowers: Maximum number of petals/ flower was found in $V_{30}(83.0)$ followed by $\mathrm{V}_{40}(80.0)$ whereas minimum from $\mathrm{V}_{44}$ (15.7) (Table 01). Variation in number of petals/ flower was also observed previously in rose (Tabassum et al., 2002; Khattak et al., 2011; Ranchana et al., 2014; Manjula, 2005; Paramagoudar, 2010) and carnation (Pralhad, 2009).

\section{Categorization of rose cultivars}

Categorization of rose cultivars on the basis of color: Color was measured by visual observation. Rose cultivars were categorized into 9 groups in accordance to their color also a wild type (Table 02). Numerous variations in color were found among 44 rose cultivars. $V_{2}, V_{3}, V_{5}, V_{8}, V_{9}, V_{12}, V_{13}, V_{19}, V_{20}$, $V_{22}, V_{24}, V_{25}, V_{29}, V_{31}, V_{32}, V_{35}, V_{38}, V_{40}$ were red to reddish, $V_{4}, V_{15}, V_{23}, V_{34}, V_{37}, V_{39}$, were yellow to yellowish, $\mathrm{V}_{10}, \mathrm{~V}_{11}, \mathrm{~V}_{17}, \mathrm{~V}_{18}, \mathrm{~V}_{27}, \mathrm{~V}_{36}, \mathrm{~V}_{41}, \mathrm{~V}_{43}, \mathrm{~V}_{44}$, were pink to pinkish, $\mathrm{V}_{16}, \mathrm{~V}_{26}, \mathrm{~V}_{21}$ were orange, $\mathrm{V}_{33}$ was off white, $V_{14} \& V_{28}$ were violet, $V_{35}$ was magenta, $V_{6} \& V_{30}$ were cream, $V_{7}$ was black and $V_{1}$ was wild type.

Categorization of rose cultivars on the basis of fragrance: Fragrances were measured by organoleptic test. On the basis of fragrance roses were divided into 3 groups viz. high, low and no fragrance (Table 03). $V_{3}, V_{4}, V_{12}, V_{13}, V_{15}, V_{16}, V_{22}, V_{25}, V_{29}, V_{32}, V_{34}, V_{35}, V_{37}, V_{38}, V_{39}, V_{40}$ were found as highly fragrant cultivars.

Categorization of rose cultivars on the basis of uses: 0 n the basis of uses were divided into 2 groups viz. cut flower and pot/bed flower (Table 04). Plant produce single flower on a stem, flower 
had compact petals with more than 5 days vase life in normal water were denoted as cut flower. $V_{3}, V_{4}$, $V_{14}, V_{16}, V_{21}, V_{23}, V_{25}, V_{26}, V_{30}$ and $V_{35}$ were found as cut flower and rest can be used as pot flower.

Table 01. Response of rose cultivars on growth and flowering attributes ${ }^{\mathrm{X}}$

\begin{tabular}{|c|c|c|c|c|c|c|c|c|c|c|c|c|c|c|c|c|}
\hline \multirow{2}{*}{$\begin{array}{l}\text { Variety }^{Y} \\
V_{1}\end{array}$} & \multicolumn{2}{|c|}{$\begin{array}{c}\text { No of } \\
\text { Shoot/plant }\end{array}$} & \multicolumn{2}{|c|}{$\begin{array}{l}\text { No. of } \\
\text { leaves }\end{array}$} & \multicolumn{2}{|c|}{$\begin{array}{l}\text { No. of } \\
\text { thorns }\end{array}$} & \multicolumn{2}{|c|}{$\begin{array}{c}\text { Leaf } \\
\text { area }\left(\mathrm{cm}^{2}\right)\end{array}$} & \multicolumn{2}{|c|}{$\begin{array}{c}\text { Chlorophyll } \\
\text { Content } \\
(\%) \\
\end{array}$} & \multicolumn{2}{|c|}{$\begin{array}{l}\text { No. of } \\
\text { flower } \\
\text { /plant }\end{array}$} & \multicolumn{2}{|c|}{$\begin{array}{l}\text { Petals } \\
\text { /flower }\end{array}$} & \multicolumn{2}{|c|}{$\begin{array}{c}\text { Days to } \\
\text { flower bud } \\
\text { initiation }\end{array}$} \\
\hline & 4.0 & $q$ & 4.0 & $\mathrm{~d}$ & 2.0 & $q$ & 24.6 & $\mathrm{u}$ & 45.3 & $j-q$ & 3.0 & 1 & 16.7 & $\mathrm{~V}-\mathrm{X}$ & 27.0 & $\mathrm{~g}$ \\
\hline$V_{2}$ & 12.0 & $\mathrm{j}$ & 3.0 & $\mathrm{e}$ & 7.0 & 1 & 35.0 & o-r & 47.3 & $i-p$ & 15.0 & $\mathrm{~d}$ & 31.0 & j & 24.0 & j \\
\hline$V_{3}$ & 9.0 & $\mathrm{~m}$ & 3.3 & $\mathrm{e}$ & 11.0 & $\mathrm{i}$ & 34.5 & $p-r$ & 59.0 & bc & 1.0 & $\mathrm{n}$ & 19.0 & st & 30.0 & $\mathrm{~d}$ \\
\hline $\mathrm{V}_{4}$ & 7.0 & 0 & 3.3 & $\mathrm{e}$ & 0.3 & $\mathrm{~s}$ & 48.1 & $\mathrm{e}$ & 52.0 & $c-k$ & 4.0 & $\mathrm{k}$ & 42.0 & $\mathrm{f}$ & 22.0 & 1 \\
\hline$V_{5}$ & 9.0 & $\mathrm{~m}$ & 5.0 & $\mathrm{C}$ & 15.0 & $\mathrm{f}$ & 30.0 & $t$ & 47.2 & $i-p$ & 3.0 & 1 & 30.0 & $\mathrm{jk}$ & 24.0 & j \\
\hline$V_{6}$ & 10.0 & 1 & 5.0 & $\mathrm{C}$ & 1.0 & $r$ & 47.1 & e & 58.9 & bc & 2.0 & $\mathrm{~m}$ & 16.3 & $w x$ & 40.0 & $a$ \\
\hline$V_{7}$ & 26.0 & $\mathrm{~d}$ & 3.0 & $\mathrm{e}$ & 11.0 & i & 36.8 & l-o & 45.0 & $\mathrm{k}-\mathrm{q}$ & 9.0 & $\mathrm{~g}$ & 19.0 & st & 18.0 & 0 \\
\hline $\mathrm{V}_{8}$ & 9.0 & $\mathrm{~m}$ & 2.0 & $\mathrm{f}$ & 3.0 & $\mathrm{p}$ & 34.7 & o-r & 50.0 & e-l & 7.3 & $\mathrm{i}$ & 21.0 & $\mathrm{qr}$ & 24.0 & $\mathrm{j}$ \\
\hline $\mathrm{V}_{9}$ & 6.0 & $\mathrm{p}$ & 5.0 & $\mathrm{c}$ & 26.0 & $\mathrm{~b}$ & 43.0 & $f-h$ & 54.0 & $b-i$ & 1.0 & $\mathrm{n}$ & 16.3 & $w x$ & 25.0 & i \\
\hline $\mathrm{V}_{10}$ & 11.0 & $\mathrm{k}$ & 3.0 & $\mathrm{e}$ & 9.0 & $\mathrm{j}$ & 41.8 & g-i & 45.0 & $\mathrm{k}-\mathrm{q}$ & 11.0 & e & 53.0 & $\mathrm{~d}$ & 36.0 & $\mathrm{~b}$ \\
\hline $\mathrm{V}_{11}$ & 23.0 & e & 3.0 & $\mathrm{e}$ & 1.0 & $r$ & 41.8 & g-i & 57.7 & $b-e$ & 20.0 & b & 33.0 & i & 20.0 & $\mathrm{~m}$ \\
\hline$V_{12}$ & 10.0 & 1 & 7.0 & $\mathrm{a}$ & 15.0 & $\mathrm{n}$ & 38.6 & $\mathrm{k}-\mathrm{m}$ & 58.9 & $\mathrm{bc}$ & 2.0 & $\mathrm{~m}$ & 36.0 & $\mathrm{~h}$ & 24.0 & j \\
\hline $\mathrm{V}_{13}$ & 4.0 & $q$ & 4.7 & $\mathrm{C}$ & 13.0 & $\mathrm{~g}$ & 39.0 & j-l & 47.1 & $i-p$ & 1.0 & $\mathrm{n}$ & 16.3 & $w x$ & 24.0 & j \\
\hline $\mathrm{V}_{14}$ & 10.0 & 1 & 4.7 & $\mathrm{c}$ & 0.3 & $\mathrm{~S}$ & 56.7 & $\mathrm{C}$ & 58.1 & $b-d$ & 5.0 & $\mathrm{j}$ & 20.0 & rs & 29.0 & e \\
\hline $\mathrm{V}_{15}$ & 9.0 & $\mathrm{~m}$ & 2.0 & $\mathrm{f}$ & 0.3 & $\mathrm{~S}$ & 31.9 & st & 35.0 & $r$ & 2.0 & $\mathrm{~m}$ & 28.0 & l & 28.0 & f \\
\hline$V_{16}$ & 8.0 & $\mathrm{n}$ & 2.0 & $\mathrm{f}$ & 24.0 & $\mathrm{c}$ & 68.4 & A & 50.5 & d-l & 4.0 & $\mathrm{k}$ & 39.0 & $\mathrm{~g}$ & 19.0 & $\mathrm{n}$ \\
\hline$V_{17}$ & 31.0 & $\mathrm{c}$ & 3.0 & $\mathrm{e}$ & 8.0 & $\mathrm{k}$ & 37.0 & $1-0$ & 44.8 & $\mathrm{k}-\mathrm{q}$ & 19.0 & $\mathrm{c}$ & 24.0 & no & 22.0 & 1 \\
\hline $\mathrm{V}_{18}$ & 8.0 & $\mathrm{n}$ & 5.0 & $\mathrm{c}$ & 2.0 & $\mathrm{q}$ & 44.6 & f & 38.7 & $\mathrm{qr}$ & 3.0 & 1 & 19.0 & st & 25.0 & $\mathrm{i}$ \\
\hline $\mathrm{V}_{19}$ & 13.0 & $\mathrm{i}$ & 4.0 & $\mathrm{~d}$ & 9.0 & $\mathrm{j}$ & 44.5 & f & 55.8 & b-h & 3.0 & 1 & 23.0 & op & 24.0 & j \\
\hline$V_{20}$ & 7.0 & o & 4.0 & $\mathrm{~d}$ & 47.0 & $\mathrm{a}$ & 35.1 & o-r & 59.2 & bc & 2.0 & $\mathrm{~m}$ & 57.0 & c & 22.0 & 1 \\
\hline$V_{21}$ & 11.0 & $\mathrm{k}$ & 3.0 & $\mathrm{e}$ & 15.0 & $\mathrm{f}$ & 67.2 & $\mathrm{a}$ & 61.2 & $a b$ & 1.0 & $\mathrm{n}$ & 22.0 & $\mathrm{pq}$ & 29.0 & $\mathrm{e}$ \\
\hline$V_{22}$ & 8.0 & $\mathrm{n}$ & 4.0 & $d$ & 5.0 & $\mathrm{n}$ & 39.5 & i-k & 47.2 & $i-p$ & 1.0 & $\mathrm{n}$ & 42.0 & f & 23.0 & $\mathrm{k}$ \\
\hline$V_{23}$ & 11.0 & $\mathrm{k}$ & 6.0 & $\mathrm{~b}$ & 7.0 & 1 & 38.2 & $\mathrm{k}-\mathrm{m}$ & 52.7 & $c-k$ & 2.0 & $\mathrm{~m}$ & 22.0 & $\mathrm{pq}$ & 29.0 & $\mathrm{e}$ \\
\hline$V_{24}$ & 14.0 & $\mathrm{~h}$ & 4.0 & $\mathrm{~d}$ & 18.0 & $\mathrm{~d}$ & 42.1 & g-i & 59.3 & bc & 4.0 & $\mathrm{k}$ & 47.0 & $\mathrm{e}$ & 23.0 & $\mathrm{k}$ \\
\hline$V_{25}$ & 13.0 & $\mathrm{i}$ & 2.0 & $\mathrm{f}$ & 3.0 & $\mathrm{p}$ & 69.5 & $\mathrm{a}$ & 56.5 & b-g & 1.0 & $\mathrm{n}$ & 39.0 & $\mathrm{~g}$ & 24.0 & j \\
\hline$V_{26}$ & 12.0 & j & 2.0 & $\mathrm{f}$ & 5.0 & $\mathrm{n}$ & 41.1 & $h-j$ & 49.6 & $f-n$ & 11.0 & $\mathrm{e}$ & 26.0 & $\mathrm{mn}$ & 23.0 & $\mathrm{k}$ \\
\hline$V_{27}$ & 32.0 & b & 4.0 & $d$ & 8.0 & $\mathrm{k}$ & 36.4 & $m-q$ & 53.2 & $c-k$ & 10.0 & $\mathrm{f}$ & 17.0 & $\mathrm{u}-\mathrm{w}$ & 24.0 & j \\
\hline$V_{28}$ & 13.0 & $\mathrm{i}$ & 4.0 & $d$ & 26.0 & $\mathrm{~b}$ & 37.6 & k-n & 41.1 & o-r & 9.0 & $\mathrm{~g}$ & 16.7 & $V-x$ & 25.0 & $\mathrm{i}$ \\
\hline$V_{29}$ & 6.0 & $\mathrm{p}$ & 4.0 & $\mathrm{~d}$ & 0.3 & $\mathrm{~S}$ & 33.3 & rs & 41.7 & $n-r$ & 3.0 & l & 22.0 & $\mathrm{pq}$ & 24.0 & j \\
\hline$V_{30}$ & 9.0 & $\mathrm{~m}$ & 3.0 & $\mathrm{e}$ & 0.3 & $\mathrm{~S}$ & 52.1 & $\mathrm{~d}$ & 53.3 & $b-j$ & 4.0 & $\mathrm{k}$ & 83.0 & $a$ & 26.0 & $\mathrm{~h}$ \\
\hline$V_{31}$ & 18.0 & f & 2.0 & $\mathrm{f}$ & 2.0 & $\mathrm{q}$ & 52.4 & $\mathrm{~d}$ & 54.0 & $b-i$ & 8.0 & $\mathrm{~h}$ & 30.0 & $\mathrm{jk}$ & 25.0 & i \\
\hline$V_{32}$ & 11.0 & $\mathrm{k}$ & 3.0 & $\mathrm{e}$ & 11.0 & i & 38.5 & $\mathrm{k}-\mathrm{m}$ & 48.6 & g-p & 8.0 & $\mathrm{~h}$ & 31.0 & j & 26.0 & $\mathrm{~h}$ \\
\hline$V_{33}$ & 4.0 & $q$ & 3.0 & $\mathrm{e}$ & 2.0 & $\mathrm{q}$ & 63.7 & $\mathrm{~b}$ & 52.0 & $\mathrm{c}-\mathrm{k}$ & 1.0 & $\mathrm{n}$ & 16.7 & $v-x$ & 25.0 & i \\
\hline$V_{34}$ & 8.0 & $\mathrm{n}$ & 3.0 & $\mathrm{e}$ & 11.0 & $\mathrm{i}$ & 33.6 & rs & 57.2 & $b-f$ & 3.0 & l & 30.0 & $\mathrm{jk}$ & 24.0 & j \\
\hline$V_{35}$ & 17.0 & $\mathrm{~g}$ & 3.0 & $\mathrm{e}$ & 2.0 & $\mathrm{q}$ & 33.2 & rs & 68.9 & $\mathrm{a}$ & 9.0 & $\mathrm{~g}$ & 16.0 & $w x$ & 25.0 & $\mathrm{i}$ \\
\hline$V_{36}$ & 42.0 & $\mathrm{a}$ & 5.0 & $\mathrm{C}$ & 4.0 & 0 & 35.6 & $n-r$ & 47.3 & $i-p$ & 9.0 & $\mathrm{~g}$ & 18.0 & tu & 25.0 & i \\
\hline$V_{37}$ & 8.0 & $\mathrm{n}$ & 5.0 & $\mathrm{c}$ & 0.3 & $S$ & 42.7 & $f-h$ & 48.0 & h-o & 2.0 & $\mathrm{~m}$ & 29.0 & $\mathrm{kl}$ & 26.0 & $\mathrm{~h}$ \\
\hline$V_{38}$ & 7.0 & o & 4.0 & $\mathrm{~d}$ & 12.0 & $\mathrm{~h}$ & 37.9 & k-n & 42.0 & $m-r$ & 2.0 & $\mathrm{~m}$ & 18.0 & tu & 24.0 & j \\
\hline$V_{39}$ & 4.0 & $\mathrm{q}$ & 4.0 & $d$ & 1.0 & $r$ & 42.8 & $f-h$ & 56.4 & b-g & 1.0 & $\mathrm{n}$ & 52.0 & $d$ & 26.0 & $\mathrm{~h}$ \\
\hline$V_{40}$ & 9.0 & $\mathrm{~m}$ & 5.0 & $\mathrm{C}$ & 17.0 & $\mathrm{e}$ & 25.3 & $\mathrm{u}$ & 43.3 & $l-q$ & 5.0 & $\mathrm{j}$ & 80.0 & b & 24.0 & j \\
\hline $\mathrm{V}_{41}$ & 6.0 & $\mathrm{p}$ & 4.7 & $c$ & 0.3 & $\mathrm{~s}$ & 30.2 & $\mathrm{t}$ & 39.9 & $p-r$ & 3.0 & 1 & 17.7 & $\mathrm{u}-\mathrm{w}$ & 24.0 & j \\
\hline$V_{42}$ & 4.0 & $q$ & 3.0 & $\mathrm{e}$ & 1.7 & $\mathrm{q}$ & 23.5 & $\mathrm{u}$ & 46.0 & $j-q$ & 1.0 & $\mathrm{n}$ & 25.0 & $\mathrm{mn}$ & 26.0 & $\mathrm{~h}$ \\
\hline$V_{43}$ & 10.0 & 1 & 4.0 & $\mathrm{~d}$ & 0.3 & $\mathrm{~s}$ & 34.0 & q-s & 49.7 & $f-m$ & 68.0 & $\mathrm{a}$ & 16.3 & $w x$ & 27.0 & $\mathrm{~g}$ \\
\hline $\mathrm{V}_{44}$ & 6.0 & $\mathrm{p}$ & 5.0 & $\mathrm{C}$ & 6.0 & $\mathrm{~m}$ & 44.1 & $f-h$ & 48.0 & h-o & 3.0 & 1 & 15.7 & $\mathrm{x}$ & 35.0 & $\mathrm{C}$ \\
\hline$L S D 0.05$ & 0.01 & & 0.6 & & 0.4 & & 2.4 & & 8.0 & & 0.3 & & 1.05 & & 0.01 & \\
\hline CV\% & 1.0 & & 9.4 & & 3.1 & & 3.7 & & 9.7 & & 2.7 & & 1.65 & & 0.01 & \\
\hline
\end{tabular}

XIn a column means having similar letter(s) are statistically identical and those having dissimilar letter(s) differ significantly as per 0.05 level of probability

YRose cultivars 
Table 02. Categorization of the rose cultivars according to color

\begin{tabular}{cll}
\hline SL. No. & \multicolumn{1}{c}{ Color } & \multicolumn{1}{c}{ Cultivars } \\
\hline 1. & Red to reddish & $\mathrm{V}_{2}, \mathrm{~V}_{3}, \mathrm{~V}_{5}, \mathrm{~V}_{8}, \mathrm{~V}_{9}, \mathrm{~V}_{12}, \mathrm{~V}_{13}, \mathrm{~V}_{19}, \mathrm{~V}_{20}, \mathrm{~V}_{22}, \mathrm{~V}_{24}, \mathrm{~V}_{25}, \mathrm{~V}_{29}, \mathrm{~V}_{31}, \mathrm{~V}_{32}, \mathrm{~V}_{35}, \mathrm{~V}_{38}, \mathrm{~V}_{40}$ \\
2. & Yellow to yellowish & $\mathrm{V}_{4}, \mathrm{~V}_{15}, \mathrm{~V}_{23}, \mathrm{~V}_{34}, \mathrm{~V}_{37}, \mathrm{~V}_{39}$ \\
3. & Pink to pinkish & $\mathrm{V}_{10}, \mathrm{~V}_{11}, \mathrm{~V}_{17}, \mathrm{~V}_{18}, \mathrm{~V}_{27}, \mathrm{~V}_{36}, \mathrm{~V}_{41}, \mathrm{~V}_{43}, \mathrm{~V}_{44}$ \\
4. & Orange & $\mathrm{V}_{16}, \mathrm{~V}_{26}, \mathrm{~V}_{21}$ \\
5. & Off white & $\mathrm{V}_{33}$ \\
6. & Violet & $\mathrm{V}_{14}, \mathrm{~V}_{28}$ \\
7. & Magenta & $\mathrm{V}_{35}$ \\
8. & Cream & $\mathrm{V}_{6}, \mathrm{~V}_{30}$ \\
9. & Black & $\mathrm{V}_{7}$ \\
10. & Wild type & $\mathrm{V}_{1}$ \\
\hline
\end{tabular}

Table 03. Categorization of the rose cultivars according to fragrance

\begin{tabular}{ccl}
\hline SL. No. & Fragrance & \multicolumn{1}{c}{ Cultivars } \\
\hline 1. & High & $\mathrm{V}_{3}, \mathrm{~V}_{4}, \mathrm{~V}_{12}, \mathrm{~V}_{13}, \mathrm{~V}_{15}, \mathrm{~V}_{16}, \mathrm{~V}_{22}, \mathrm{~V}_{25}, \mathrm{~V}_{29}, \mathrm{~V}_{32}, \mathrm{~V}_{34}, \mathrm{~V}_{35}, \mathrm{~V}_{37}, \mathrm{~V}_{38}, \mathrm{~V}_{39}, \mathrm{~V}_{40}$ \\
2. & Low & $\mathrm{V}_{2}, \mathrm{~V}_{8}, \mathrm{~V}_{10}, \mathrm{~V}_{11}, \mathrm{~V}_{14}, \mathrm{~V}_{20}, \mathrm{~V}_{21}, \mathrm{~V}_{24}, \mathrm{~V}_{26}, \mathrm{~V}_{27}, \mathrm{~V}_{28}, \mathrm{~V}_{31}, \mathrm{~V}_{33}, \mathrm{~V}_{36}, \mathrm{~V}_{41}, \mathrm{~V}_{42}, \mathrm{~V}_{44}$ \\
3. & No & $\mathrm{V}_{5}, \mathrm{~V}_{6}, \mathrm{~V}_{7}, \mathrm{~V}_{9}, \mathrm{~V}_{17}, \mathrm{~V}_{18}, \mathrm{~V}_{19}, \mathrm{~V}_{23}, \mathrm{~V}_{30}, \mathrm{~V}_{43}$ \\
4. & Wild type & $\mathrm{V}_{1}$ \\
\hline
\end{tabular}

Table 04. Categorization of the rose cultivars according to usage

\begin{tabular}{ccl}
\hline SL. No. & Usage & \multicolumn{1}{c}{ Cultivars } \\
\hline 1. & Cut flower & $\mathrm{V}_{3}, \mathrm{~V}_{4}, \mathrm{~V}_{14}, \mathrm{~V}_{16}, \mathrm{~V}_{21}, \mathrm{~V}_{23}, \mathrm{~V}_{25}, \mathrm{~V}_{26}, \mathrm{~V}_{30}$ and $\mathrm{V}_{35}$ \\
2. & Pot/bed flower & $\mathrm{V}_{2}, \mathrm{~V}_{5}, \mathrm{~V}_{6}, \mathrm{~V}_{7}, \mathrm{~V}_{8}, \mathrm{~V}_{9}, \mathrm{~V}_{10}, \mathrm{~V}_{11}, \mathrm{~V}_{12}, \mathrm{~V}_{13}, \mathrm{~V}_{15}, \mathrm{~V}_{17}, \mathrm{~V}_{18}, \mathrm{~V}_{19}, \mathrm{~V}_{20}, \mathrm{~V}_{22}, \mathrm{~V}_{24}, \mathrm{~V}_{27}, \mathrm{~V}_{28}$, \\
3. & Wild type & $\mathrm{V}_{29}, \mathrm{~V}_{31}, \mathrm{~V}_{32}, \mathrm{~V}_{33}, \mathrm{~V}_{34}, \mathrm{~V}_{36}, \mathrm{~V}_{37}, \mathrm{~V}_{38}, \mathrm{~V}_{39}, \mathrm{~V}_{40}, \mathrm{~V}_{41}, \mathrm{~V}_{42}, \mathrm{~V}_{43}$ and $\mathrm{V}_{44}$ \\
\hline
\end{tabular}

\section{Conclusion}

Rose cultivars varied significantly different studied parameters like number of shoots/plant, leaf area, chlorophyll content, number of flower/plant and petals per flower also varied in color, fragrance and usages. Tajmahal $\left(\mathrm{V}_{3}\right)$, Yellow star $\left(\mathrm{V}_{4}\right)$, Lavender gold $\left(\mathrm{V}_{14}\right)$, Compassion $\left(\mathrm{V}_{16}\right)$, SAU hero $\left(\mathrm{V}_{21}\right)$, Yellow gold $\left(V_{23}\right)$, Sleepy moon $\left(V_{25}\right)$, Sweet doll $\left(V_{26}\right)$, Chrysanthemum rose $\left(V_{30}\right)$, Sweet sakata $\left(V_{35}\right)$ can be used for commercial cut flowers production in farmer's field. But further trial is needed using the cut rose cultivars only. Rest of the cultivars can be produced as pot/bed flowers in accordance of the user choice. Breeding programmes of the rose focus on improvements of various characteristics to enhance ornamental value including color, size, form, fragrance of the bloom and plant response to the environment. Growth and yield performance evaluation and morphological categorization of these forty four rose cultivars by color, fragrance and usage may help to the breeder to select the parent for the improvement of the rose.

\section{References}

[1]. Ahmad, F., Khan, O., Sarwar, S., Hussain, A. and Ahmad, S. (2007). Performance evaluation of tomato cultivars at high altitude. Sarhad J. Agric. 23(3): 581-585.

[2]. Ahmad, I., Khalid, M. S., Khan, M. A. and Saleem, M. (2011). Morpho-physiological comparison of cut rose cultivars grown in two production systems. Pak. J. Bot. 43(6): 2885-2890. 
[3]. Ahsan, M. K., Mehraj, H., Hussain, M. S., Rahman, M. M. and Jamal Uddin, A. F. M. (2014). Study on growth and yield of three promising strawberry cultivars in Bangladesh. Int. J. Bus. Soc. Sci. Res. 1(3): 205-208.

[4]. Biswas, M., Sarkar, D. R., Asif, M. I., Sikder, R. K., Mehraj, H. and Jamal Uddin, A. F. M. (2014). Comparison of growth and yield characteristics of BARI tomato varieties. J. Biosci. Agric. Res. 3(1): 1-7.

[5]. Cherri-Martin, M., Jullien, F., Heizmann, P. and Baudino, S. (2007). Fragrance heritability in hybrid tea roses. Sci. Hortic. 113: 177-181.

[6]. Chobe, R. R., Pachankar, P. B. and Warde, S. D. (2010). Performence of different cultivars of gerbera under polyhouse condition. The Asian Journal of Horticulture 5(2): 333-335.

[7]. Datta, J. K., Sikdar, M. S., Banerjee, A. and Mondal, N. K. (2011). Screening of mustard varieties under combined dose of fertilizers and subsequent soil health and biodiversity in old alluvial soli of Burdwan, West Bengal, India. World Applied Sciences J. 13(2): 217-225.

[8]. Gautam, U. S., Negi, R. S., Rajesh, S., Kaushik, S. S. and Alka, S. (2013). Participatory evaluation of tomato varieties for commercial cultivation during rainy season under Kaymore Plateau and Satpura Hills - Agro-climatic zone of Madhya Pradesh. J. of Agril. Sci. 5(4): 238-241.

[9]. Gharge, C. P., Angadi, S. G., Basavaraj, N., Patil, A. A., Biradar, M. S. and Mummigatti, U. V. (2011). Performance of Standard carnatin varieties under naturally ventillated polyhouse. Karnataka J. Agric. Sci. 24(4): 487-489.

[10]. Gomez, K. A. and Gomez, A. A. (1984). Statistical Procedures for Agricultural Research. 2nd edn. John Wiley and Sons. New York. p. 680.

[11]. Hammad, I. (2009). Genetic variation among Bougainvillea glabra cultivars (Nyctaginaceae) detected by rapd markers and isozymes patterns. Res. J. Agriculture and Biological Sci. 5(1): 6371.

[12]. Hossain, S., Jolly, S. N., Parvin, S., Mehraj, H. and Jamal Uddin, A. F. M. (2015). Performance on growth and flowering of sixteen hybrid gerbera cultivars. Int. J. Bus. Soc. Sci. Res. 3(2): 87-92.

[13]. Hossan, M. J., Islam, M. S., Ahsan, M. K., Mehraj, H. and Jamal Uddin, A. F. M. (2013). Growth and yield performance of strawberry germplasm at Sher-e-Bangla Agricultural University. J. Expt. Biosci. 4(1): 89-92.

[14]. Jamal Uddin, A. F. M., Faruq, M. A. A., Roni, M. Z. K., Taufique, T. and Mehraj, H. (2014c). Growth and yield performance of four pumpkin (Cucurbita moschata) lines. Int. J. Bus. Soc. Sci. Res. 2(2): 113-115.

[15]. Jamal Uddin, A. F. M., Islam, M. S., Mehraj, H., Roni, M. Z. K. and Shahrin, S. (2013). An evaluation of some Japanese lisianthus (Eustoma grandiflorum) varieties grown in Bangladesh. The Agriculturists 11(1): 56-60.

[16]. Jamal Uddin, A. F. M., Tahidul, M. I., Chowdhury, M. S. N., Shiam, I. H. and Mehraj, H. (2014b). Evaluation of bottle gourd (Lagenaria siceraria) to growth and yield. Int. J. Biosci. 5(12): 7-11.

[17]. Jamal Uddin, A.F.M., H. Mehraj, T. Taufique, A.F. Ona and S. Parvin. (2014a). Foliar application of gibberellic acid on growth and flowering of gerbera cultivars. J. Biosci. Agric. Res. 2(1): 52-58.

[18]. Khattak, A. M., Dawar, S. H., Khan, M. A. and Razaq, A. (2011). Effect of summer pruning on the quality and performance of rose cultivars. Sarhad J. Agric. 27(1): 27-31.

[19]. Kumari, A., Patel, K. S. and Nayee, D. D. (2010). Evaluation of different cultivars of gerbera (Gerbera jamisonii bolus ex hooker F.) for growth yield and quality grown under fan and pad cooled green house condition. The Asian Journal of Horticulture 5(2): 309-310.

[20]. Mahanta, S., Talukdar, M. C. and Sarma, B. (2003). Evaluation of black centred gerbera cultivars under polyhouse. Nation. Symp. Recent Adv. Indian Floric. Trichur, 12-14 November, Proc. Indian Soc. Orna. Hort., 12-14 Nov., pp. 175-177.

[21]. Mahmood, M. A., Ahmad, N. and Khan, M. S. A. (2013). Comparative evaluation of growth, yield and quality characteristics of various gerbera (Gerbera jamesonii L.) cultivars under protected condition. Journal of Ornamental Plants 3(4): 235-241.

[22]. Maitra, S. and Roychowdhury, N. (2013). Performance of diferent standard carnaton (Dianthus caryophyllus L.) cultvars in the plains of West Bengal, India. International Journal of Bio-resource and Stress Management 4(3): 395-399.

[23]. Manjula, Ms. G. (2005). Performance of rose cultivars under naturally ventilated polyhouse. MS. Thesis. University of Agricultural Sciences, Dharwad. India. pp. 29-37. 
[24]. Mehraj, H., Ahsan, M. K., Mahmud, M. F., Hussain, M. S. and Jamal Uddin, A. F. M. (2014a). characterization of three strawberry germplasm at Sher-e-Bangla Agricultural University. Int. J. Bus., Soc. and Sci. Res. 1(3): 134-138.

[25]. Mehraj, H., Mutahera, S., Roni, M. Z. K., Nahiyan, A. S. M. and Jamal Uddin, A. F. M. (2014d). Performance assessment of twenty tomato varieties for summer cultivation in Bangladesh. J. Sci. Technol. Environ. Inform. 1(1): 45-53.

[26]. Mehraj, H., Shikha, K. J., Nusrat, A., Shiam, I. H. and Jamal Uddin, A. F. M. (2014e). Growth and flowering behaviour of dendrobium cultivars. J. Biosci. Agric. Res. 2(2): 90-95.

[27]. Mehraj, H., Tamima, M. H., Chowdhury, M .S. N., Ferdous, M. H. and Jamal Uddin, A. F. M. (2014c). Study on Morpho-physiological characteristics and yield performance of four chilli lines. J. Biosci. Agric. Res. 2(1): 1-7.

[28]. Mehraj, H., Taufique, T., Ona, A. F., Nusrat, A. and Jamal Uddin, A. F. M. (2014b). Performance of gerbera cultivars under different wavelengths of solar spectrum. Journal of Bangladesh Academy of Sciences 38(1): 27-37.

[29]. Moniruzzaman, M., Rahman, S. M. L., Kibria, M.G., Rahman, M. A. and Kaisar, M. O. (2007). Performances of vegetable french bean as influenced by varieties and sowing dates in rabi season. Int. J. Sustain. Crop Prod. 2(5): 69-73.

[30]. Munikrishnappa, P. M., Patil, A. A., Patil, V. S., PatiL, B. N., Channappagoudar, B. B. and Alloli, T. B. (2013). Studies on the growth and yield parameters of different genotypes of China aster (Callistephus chinensis Nees.). Karnataka J. Agric. Sci. 26(1): 107-110.

[31]. Nadeem, M., Khan, M. A., Riaz, A. and Ahmad. (2011). Evaluation of growth and flowering potential of Rosa hybrida cultivars under Faisalabad climatic conditions. Pak. J. Agri. Sci. 48(4): 283-288.

[32]. Nahiyan, A. S. M., Momena, K., Mehraj, H., Shiam, I. H., Jamal Uddin, A. F. M. and Rahman, L. (2014). Genetic diversity of sixteen tomato varieties grown at Sher-e-Bangla Agricultural University. Int. J. Bus. Soc. Sci. Res. 2(1): 39-44.

[33]. Olaniyi, J. O., Akanbi, W. B., Adejumo, T. A. and Akande, O. G. (2010). Growth, fruit yield and nutritional quality of tomato varieties. African J. Food Sci. 4(6): 398-402.

[34]. Paramagoudar, P. (2010). Performance of Dutch roses under polyhouse. University of Agricultural Sciences, Dharwad. India. pp. 27-33.

[35]. Pattanashetti, C. N. (2009). Evaluation of gerbera cultivars under protected conditions. MS. Thesis. University of Agricultural Sciences, Dharwad. India. pp. 37-42.

[36]. Pralhad, G. C. (2009). Evaluation of carnation (Dianthus caryophyllus L.) varieties under greenhouse condition. University of Agricultural Sciences, Dharwad. India. pp. 25-55.

[37]. Ramzan, A., Hanif, M., and Tariq, S. 2014. Performance of Rosa Hybrida cultivars under agroclimatic conditions of Islamabad, Pakistan. J. Agric. Res. 52(1):153-159.

[38]. Ranchana, P., Selvaraj, N., Jawaharlal, M., Kumar, C. S. R., and Anita, B. (2014). Cut rose varieties suitable for organic farming system in Nilgiris, Tamil Nadu. J. Biol. Chem. Research. 31(1): 104108.

[39]. Reddy, B. S., Kulkarni, B. S., Manjunath, H. K. and Shiragur, M. (2003). Performance of gerbera cultivars under naturally ventilated greenhouse. Paper presented in All India Seminar on Potential and Prospects for Protective Cultivation, pp. 91-92.

[40]. Sankar, M., Sreelatha, U., Rajeevan, P. K., Bhaskar, J. and Krishnan, S. (2003). Varietal evaluation of gerbera (Gerbera jamesonii Bolus) under low cost greenhouse. Nation. Symp. Recent Adv. Indian Floric. Trichur, 12-14 November, Proc. Indian Soc. Orna. Hort., 12-14 Nov., pp. 172-174.

[41]. Shruti, W. and Gajbhiye, R. P. (2013). Evaluation of gerbera varieties for growth and flowering under shadenet. International Journal of Horticulture 3(9): 42-45.

[42]. Singh, A. K., Singh, D. K., Singh, B., Punetha, S. and Rai, D. (2013). Evaluation of Carnation (Dianthus caryophyllus L.) Varieties under naturally ventilated greenhouse in mid hills of Kumaon Himalaya. African Journal of Agricultural Research 8(29): 4111-4114.

[43]. Singh, K. P. and Ramachandran, N. (2002). Comparison of greenhouses having natural ventilation and fan and pad evaporative cooling systems for gerbera production. J. Orna. Hort. 5(2): 15-19.

[44]. Tabassum, R., Ghaffoor, A., Waseem, K. and Nadeem, M. A. (2002). Evaluation of rose cultivars as cut flower production. Asian Journal of plant science 1(6):668-669.

[45]. Tarannum, Ms. and Naik, B. H. (2014). Performance of carnation (Dianthus caryophyllus L.) genotypes for qualitative and quantitative parameter to assess genetic variability among 
genotypes. American International Journal of Research in Formal, Applied \& Natural Sciences 5(1): 96-101.

[46]. Zosiamliana, J. H., Reddy, G. S. N. and Rymbai, H. (2012). Growth, flowering and yield characters of some cultivars of China aster (Callistephus chinensis Ness.). J. Nat. Prod. Plant Resour. 2(2): 302-305. 


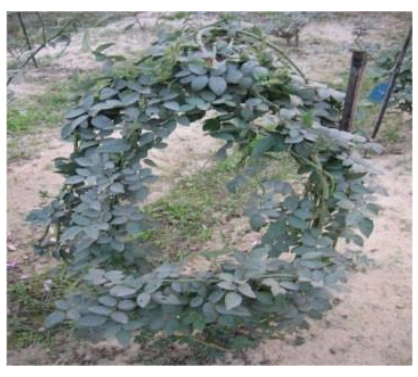

$\mathrm{V}_{1}$

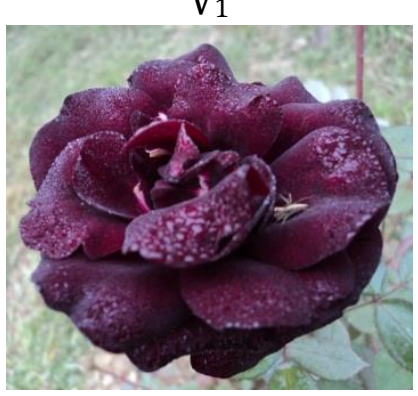

$\mathrm{V}_{7}$

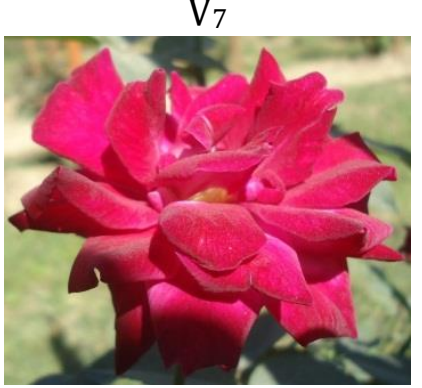

$\mathrm{V}_{13}$

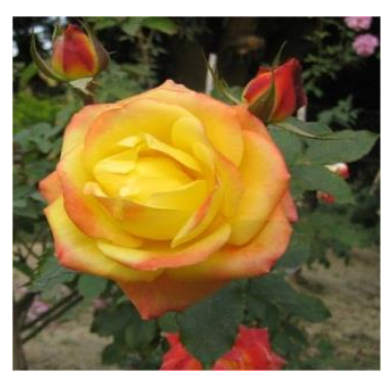

$\mathrm{V}_{2}$

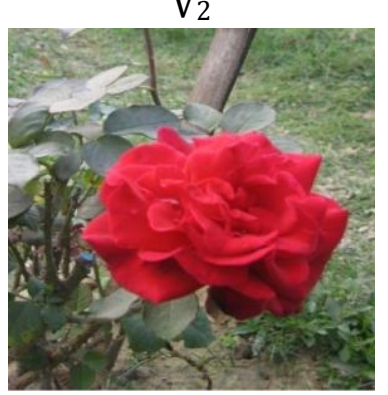

$\mathrm{V}_{8}$

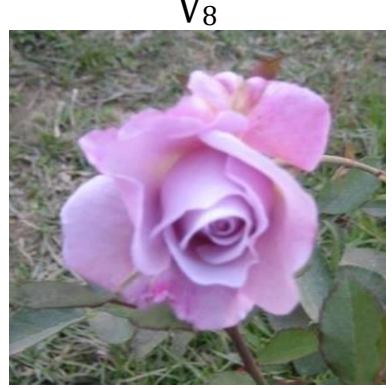

$\mathrm{V}_{14}$

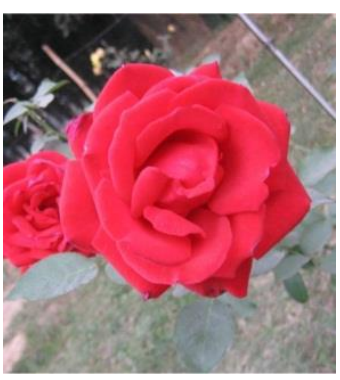

$\mathrm{V}_{3}$

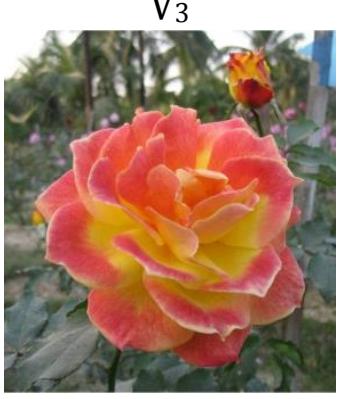

$\mathrm{V}_{9}$

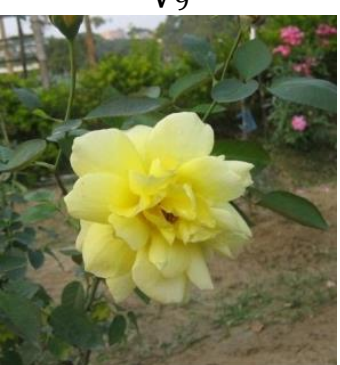

$\mathrm{V}_{15}$

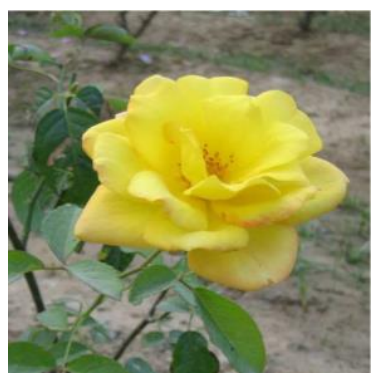

$\mathrm{V}_{4}$

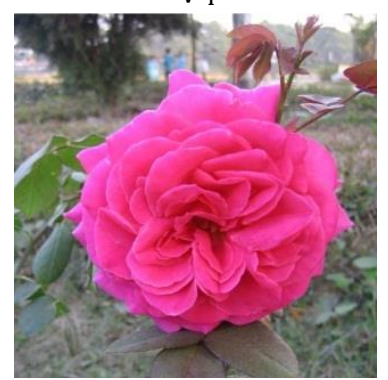

$\mathrm{V}_{10}$

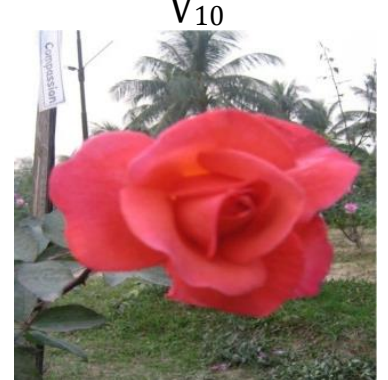

$\mathrm{V}_{16}$

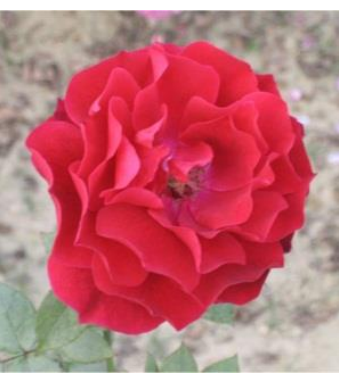

$\mathrm{V}_{5}$

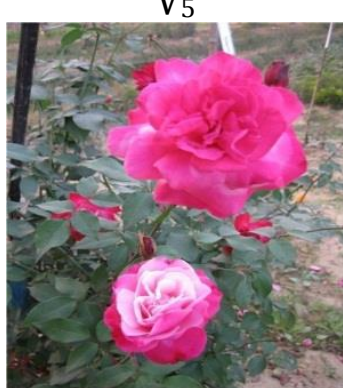

$\mathrm{V}_{11}$

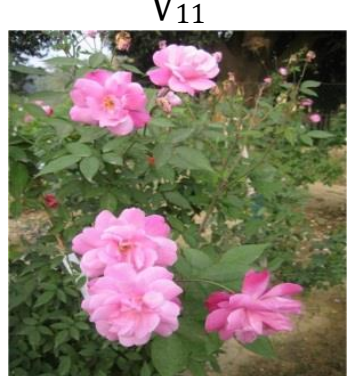

$\mathrm{V}_{17}$

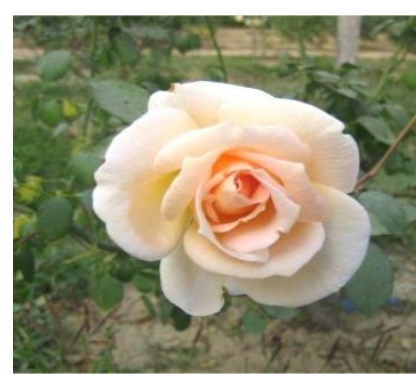

$\mathrm{V}_{6}$

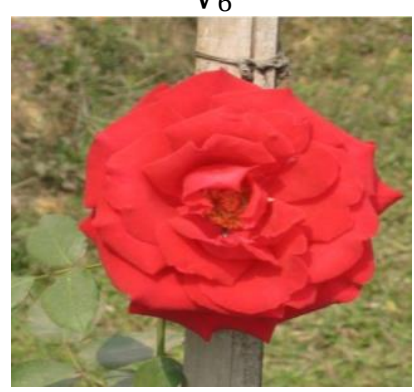

$\mathrm{V}_{12}$

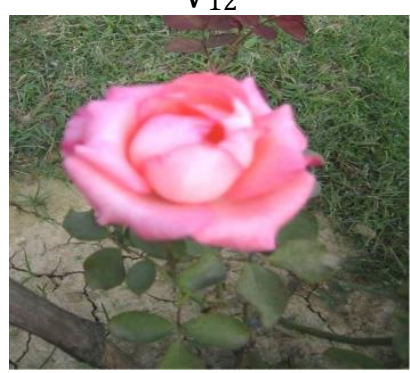

$\mathrm{V}_{18}$

Plate 01. Pictorial views of forty four rose cultivars (continued) 


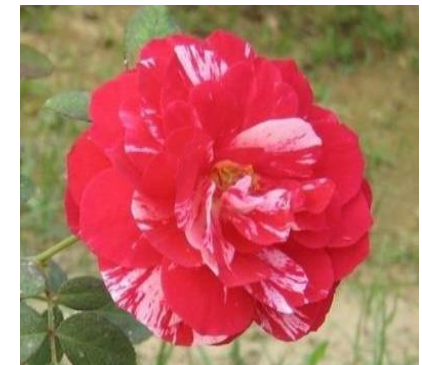

$\mathrm{V}_{19}$

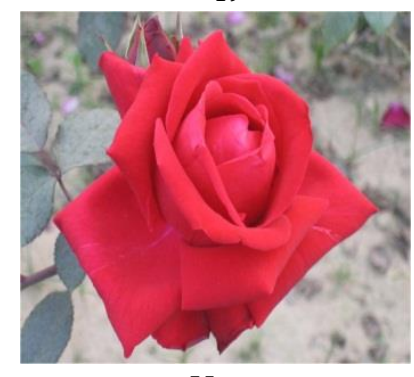

$\mathrm{V}_{25}$

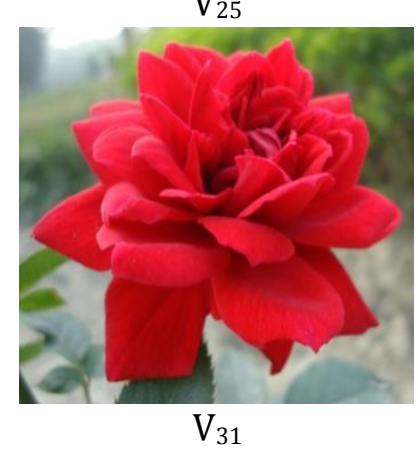

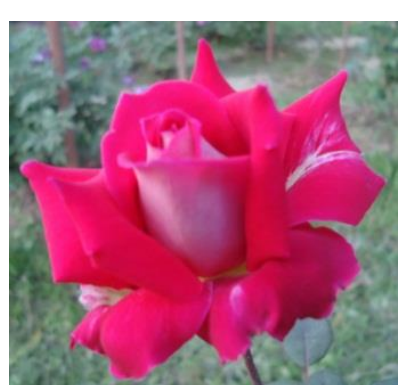

$\mathrm{V}_{20}$

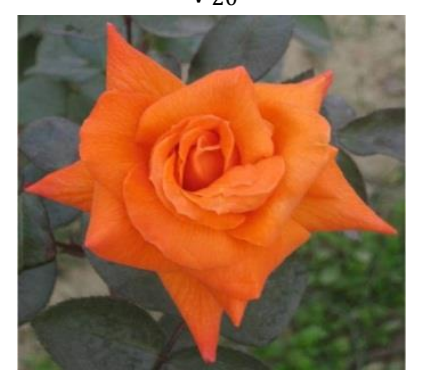

$\mathrm{V}_{26}$

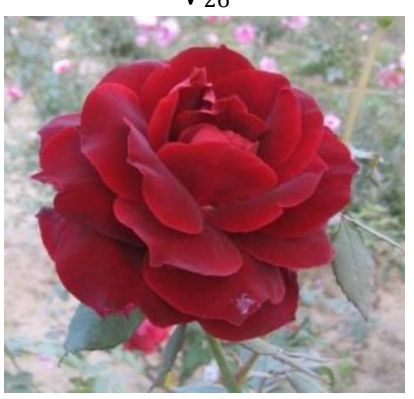

$\mathrm{V}_{32}$

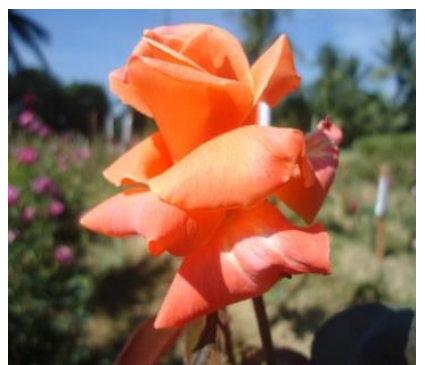

$\mathrm{V}_{21}$

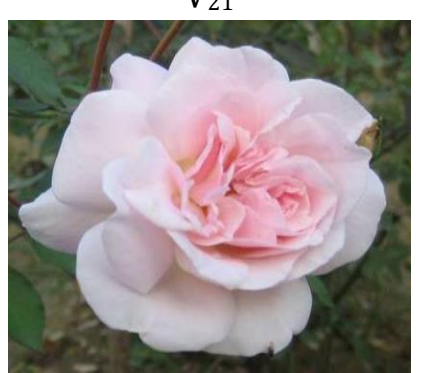

$\mathrm{V}_{27}$

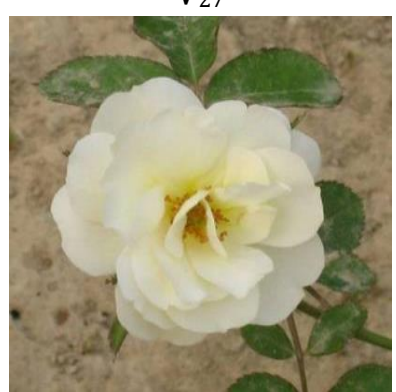

$\mathrm{V}_{33}$

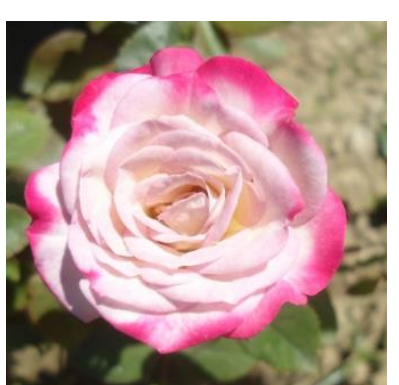

$\mathrm{V}_{22}$

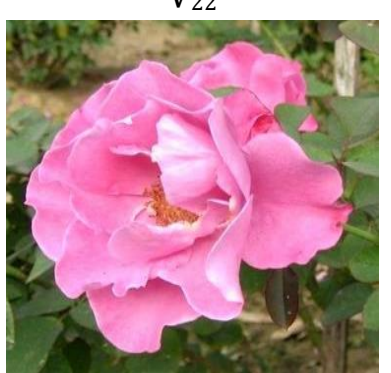

$\mathrm{V}_{28}$

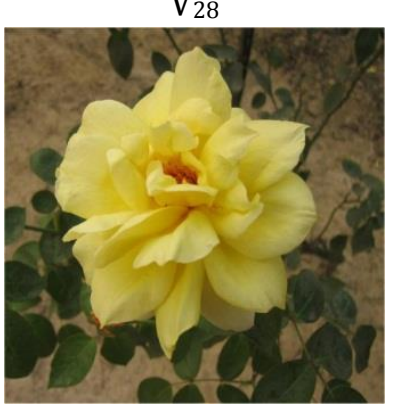

$\mathrm{V}_{34}$

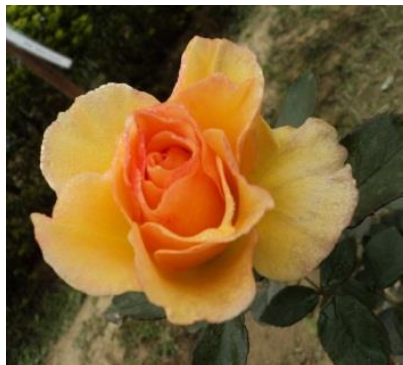

$\mathrm{V}_{23}$

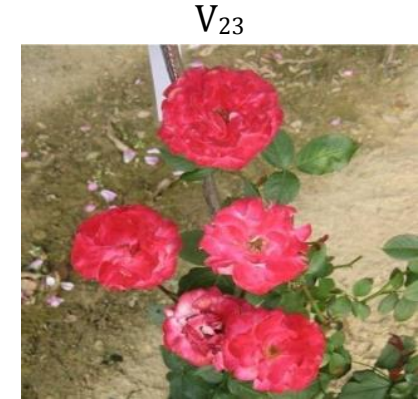

$\mathrm{V}_{29}$

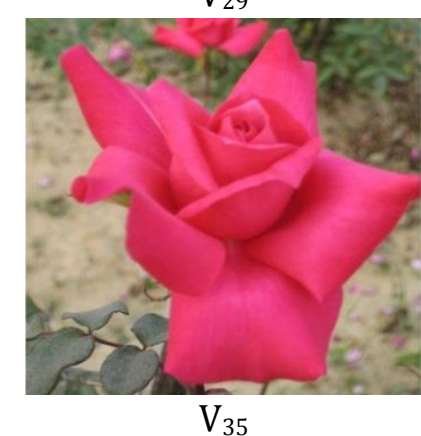

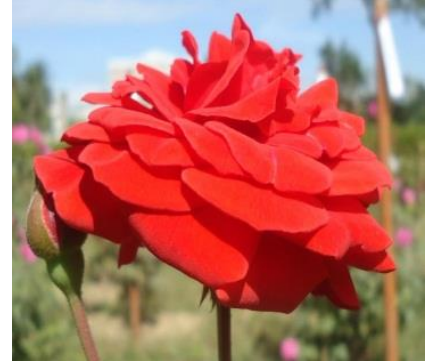

$\mathrm{V}_{24}$
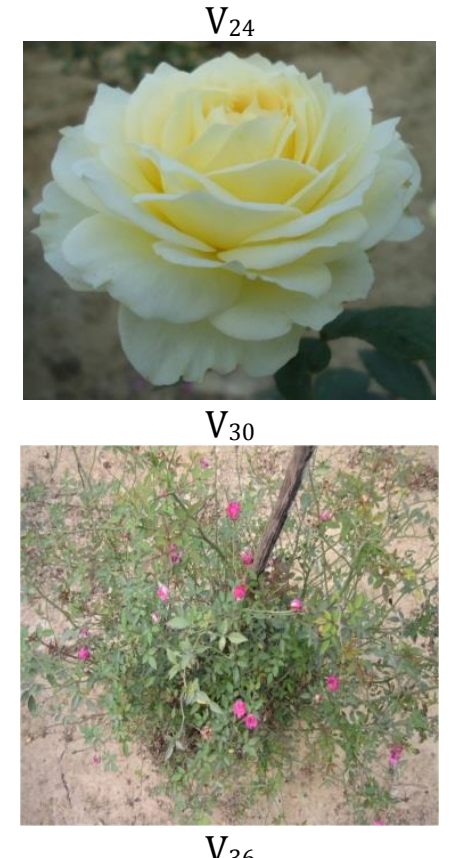

$\mathrm{V}_{36}$

Plate 01. Pictorial views of forty four rose cultivars (continued) 


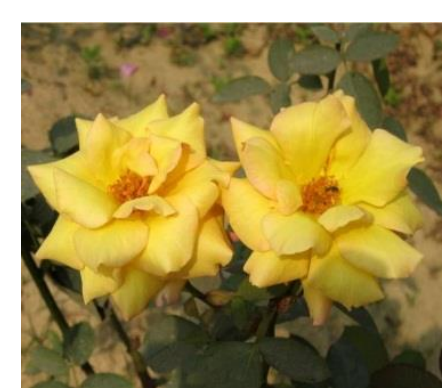

$\mathrm{V}_{37}$

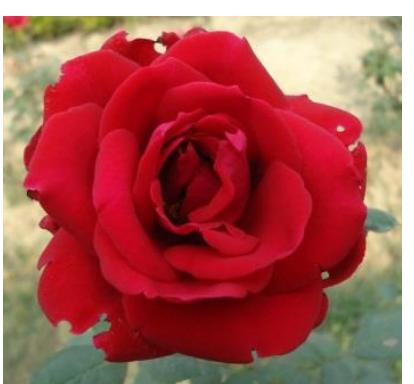

$\mathrm{V}_{38}$

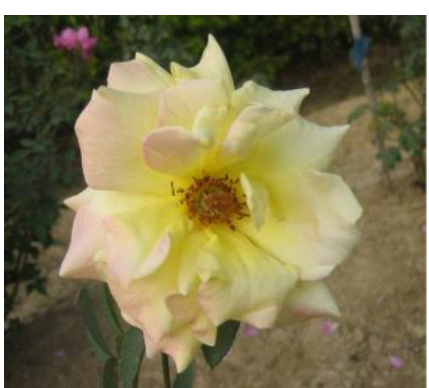

$\mathrm{V}_{39}$

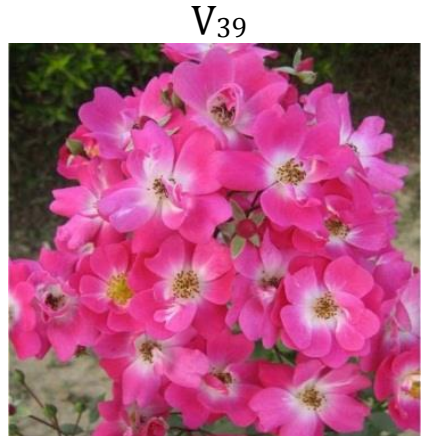

$\mathrm{V}_{43}$

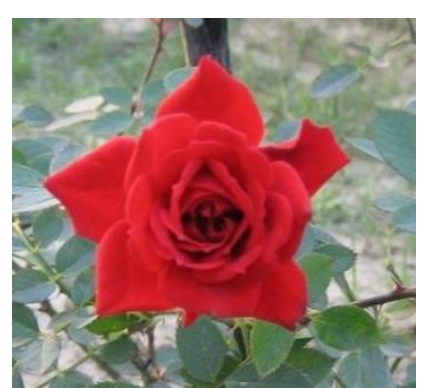

$\mathrm{V}_{40}$

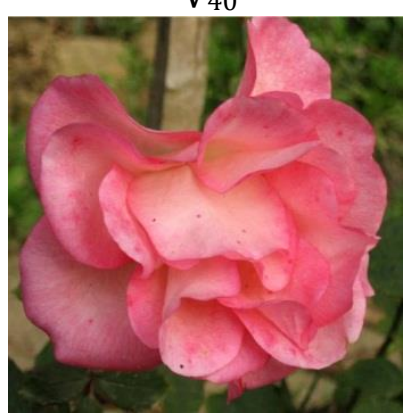

$\mathrm{V}_{44}$

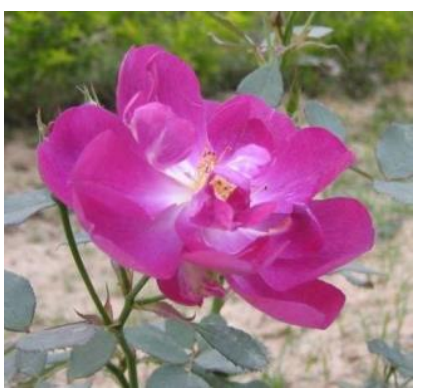

$\mathrm{V}_{41}$

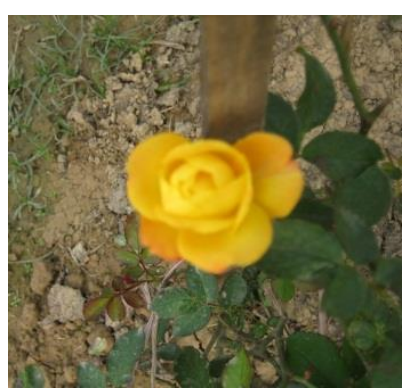

$\mathrm{V}_{42}$

Plate 01. Pictorial views of forty four rose cultivars

$\mathrm{V}_{1}$ : Wild rose; $\mathrm{V}_{2}$ : Crazy love bicolor; $\mathrm{V}_{3}$ : Tajmahal; $\mathrm{V}_{4}$ : Yellow star; $\mathrm{V}_{5}$ : Ekuse lal; $\mathrm{V}_{6}$ : Afrodita; $\mathrm{V}_{7}$ : black divorce; $\mathrm{V}_{8}$ : Attarcted red; $\mathrm{V}_{9}$ : Missing love; $\mathrm{V}_{10}$ : Pinky smile; $\mathrm{V}_{11}$ : Sweet love; $\mathrm{V}_{12}$ : Red baby; $\mathrm{V}_{13}$ : Star fire; $\mathrm{V}_{14}$ : Lavender gold; $\mathrm{V}_{15}$ : Lemon star; $\mathrm{V}_{16}$ : Compassion; $\mathrm{V}_{17}$ : Charming lady; $\mathrm{V}_{18}$ : Dream bangla; $\mathrm{V}_{19}$ : Printed moon; $\mathrm{V}_{20}$ : Fire and ice; $\mathrm{V}_{21}$ : $\mathrm{SAU}_{2}$ hero $\mathrm{V}_{22}$ : SAU prince; $\mathrm{V}_{23}$ : Yellow gold; $\mathrm{V}_{24}$ : Ocean heart; $\mathrm{V}_{25}$ : Sleepy moon; $\mathrm{V}_{26}$ : Sweet doll; $\mathrm{V}_{27}$ : Moon light; $\mathrm{V}_{28}$ : Pain blue; $\mathrm{V}_{29}$ : Ruin night; $\mathrm{V}_{30}$ : Chrysanthemum rose; $\mathrm{V}_{31}$ : Mystery love; $\mathrm{V}_{32}$ : SAU king; $\mathrm{V}_{33}$ : Ice barg; $\mathrm{V}_{34}$ : Yellow erecta; $\mathrm{V}_{35}$ : Sweet sakata; $\mathrm{V}_{36}$ : Anindo kun hazari; $\mathrm{V}_{37}$ : Yellow papiliom; $\mathrm{V}_{38}$ : Red eye; $\mathrm{V}_{39}$ : Sympathy; $\mathrm{V}_{40}$ : Mini master; $\mathrm{V}_{41}$ : Pretty girl; $\mathrm{V}_{42}$ : Sad 23; $\mathrm{V}_{43}$ : Aranchan and $\mathrm{V}_{44}$ : Sweet mum 